\title{
Compromiso cardiovascular secundario a tumor carcinoide metastásico
}

Cardiovascular compromise secondary to metastatic carcinoid tumor

Mateo Mejía-Zuluaga' ORCID, Tatiana María Bedoya-Jaramillo ${ }^{2}$ ORCID, Néstor Ricardo Duarte-
Suárez $^{3}$ ORCID, Sebastián Naranjo-Restrepo ${ }^{4}$ ORCID, Mauricio Duque-Ramírez ${ }^{5}$ ORCID

Fecha correspondencia: Recibido: marzo 4 de 2020. Revisado: septiembre 21 de 2020. Aceptado: octubre 20 de 2020.

Forma de citar:

Mejía-Zuluaga M, BedoyaJaramillo TM, Duarte-Suárez NR,Naranjo-Restrepo S, Duque-Ramírez S. Compromiso cardiovascular secundario a tumor carcinoide metastásico. Rev CES Med. 2020; 34(3): 228-235.

\section{Open access}

(C) Derecho de autor

Licencia creative commons

Ética de publicaciones

Revisión por pares

Gestión por Open Journal System DOl: http://dx.doi.org/10.21615/ cesmedicina.34.3.7

ISSN 0120-8705

e-ISSN 2215-9177
Comparte

\section{ค曰G.}

\section{Resumen}

Los tumores carcinoides son neoplasias pertenecientes a la familia de tumores neuroendocrinos y presentan baja incidencia en la población en general; sin embargo, hasta el $50 \%$ pueden debutar con compromiso cardiovascular. Se presenta el caso de un paciente, sin antecedentes cardiovasculares quien presentó signos y síntomas sugestivos de falla cardiaca derecha. Se le realizó ecocardiografía transtorácica que evidenció compromiso de ambas válvulas cardíacas derechas por tumor neuroendocrino metastásico, llevando al diagnóstico de sindrome carcinoide. Con el fin de tener presente dicha entidad en la práctica diaria de la cardiología clínica se revisan datos claves de abordaje diagnóstico y terapéutico.

Palabras clave: Cardiopatía carcinoide; Tumor carcinoide; Síndrome carcinoide maligno.

\begin{abstract}
Carcinoid tumors are neoplasms belonging to the neuroendocrine tumor family and have a low incidence in the general population; however, up to $50 \%$ may debut with cardiovascular involvement. We present the case of a patient, without a cardiovascular history, who presented signs and symptoms suggestive of right-sided heart failure. A transthoracic echocardiography was performed, which evidenced the involvement of both right heart valves by a metastatic neuroendocrine tumor, leading to the diagnosis of carcinoid syndrome. In order to take this entity into account in the daily practice of clinical cardiology, key data of diagnostic and therapeutic approach are reviewed.
\end{abstract}

Keywords: Carcinoid heart disease; Carcinoid tumor; Malignant Carcinoid syndrome.

\section{Introducción}

Los tumores carcinoides son neoplasias poco frecuentes, tienen su origen en el sistema neuroendocrino y se caracterizan por células del sistema nervioso o endocrino. Su localización más común es en el sistema gastrointestinal, específicamente en el intestino delgado. Estos tumores son 


\section{Sobre los autores:}

1. Internista - Cardiólogo.

Clínica Medellín de

Occidente.

\author{
2. Residente de \\ Anestesiología, Universidad \\ CES.
}

3. Internista - Cardiólogo-

Ecocardiografista. Clínica

CES.

\section{Internista - Cardiólogo. \\ Clínica CES, Hospital \\ General de Medellín. \\ Docente Medicina interna y \\ Cardiología Universidad CES.}

5. Internista - Cardiólogo

- Electrofisiólogo. CES

cardiología: Clínica CES y

Sede San Diego. Profesor

Titular Universidad CES-

Jefe de postgrado de

Electrofisiología y jefe de

postgrado de Cardiología

Universidad CES. Grupo de

investigación: Enfermedades

Del Corazón.

La mayoría de síntomas están explicados según las sustancias secretadas: serotonina, histamina, bradiquinina y prostaglandinas, que son las causantes del enrojecimiento, la diarrea, las nauseas, el dolor abdominal, las crisis broncoconstrictivas e incluso la cardiopatía restrictiva. de crecimiento lento, teniendo una edad de presentación alrededor de la séptima década de la vida; sin embargo, no es infrecuente encontrar al momento del diagnóstico, compromiso metastásico, principalmente en hígado, pulmón y corazón (1).

La mayoría de síntomas estan explicados según las sustancias secretadas: serotonina, histamina, bradiquinina y prostaglandinas, que son las causantes del enrojecimiento, la diarrea, las nauseas, el dolor abdominal, las crisis broncoconstrictivas e incluso la cardiopatía restrictiva presentada por los pacientes $(1,2)$.

El compromiso cardiovascular no es infrecuente: está presente hasta en el $50 \%$ de los pacientes con síndrome carcinoide, incluso, puede ser la forma de presentación del tumor hasta en un $20 \%$ de los casos $(1,3)$. La afección se da principalmente en las cavidades derechas del corazón, siendo raro el compromiso de cavidades izquierdas (4-7).

Se presenta el caso de un paciente con tumor carcinoide metastásico y compromiso cardiovascular secundario.

\section{Caso clínico}

Se trataba de un hombre de 63 años quien estaba en seguimiento ambulatorio por Endocrinología por tumor neuroendocrino de bajo grado, con síndrome carcinoide asociado, presencia de lesiones metastásicas a hígado y peritoneo, con localización del tumor primario en intestino delgado, identificado por octreoscan, y en tratamiento con somatostatina y lanreotide de reciente inicio.

Consultó a una institución de tercer nivel por cuadro clínico de un mes de evolución consistente en edema de miembros inferiores, ortopnea y disnea paroxística nocturna. No tenía antecedente cardiovascular de ningún tipo, negaba haber presentado episodios de angina, disnea o palpitaciones.

En el abordaje de su falla cardíaca se solicitó ecocardiografía transtorácica que evidenció compromiso de ambas válvulas cardíacas derechas: válvula tricuspídea con engrosamiento y esclerosis de sus valvas y restricción en su movilidad, especialmente de las valvas anterior y septal, causando estenosis severa (gradiente máximo $17 \mathrm{~mm} / \mathrm{Hg}$, gradiente medio $7 \mathrm{~mm} / \mathrm{Hg}$, área valvular por tiempo de hemipresión de $1,0 \mathrm{~cm}^{2}$ ) e insuficiencia moderada a severa (figura 1). La válvula pulmonar presentaba engrosamiento de sus valvas, estenosis leve, sin insuficiencia (figura 2), asociado a hipertensión pulmonar moderada con una presión sistólica de la arteria pulmonar de $61 \mathrm{~mm} / \mathrm{Hg}$. Se evidenció, además, pericardio visceral levemente engrosado, con líquido pericárdico en cantidad aproximada de 250 cc globalmente distribuido, sin signos de repercusión hemodinámica ni de constricción. Finalmente, en vista ecocardiográfica subcostal modificada se observaron metástasis hepáticas (figura 3). Respecto a las cavidades izquierdas no se reportó ninguna alteración de importancia, presentaba una función sistólica normal con fracción de eyección del 60 \%.

Se inició manejo de falla cardíaca con terapia diurética, presentando notable mejoría, motivo por el que se definió continuar manejo médico conservador, realizar control ecocardiografíco una vez se completaran tres meses de tratamiento con análogos de somatostatina, y según la evolución, definir la necesidad de intervención quirúrgica valvular. 
Septiembre - diciembre de 2020 - Pág 230

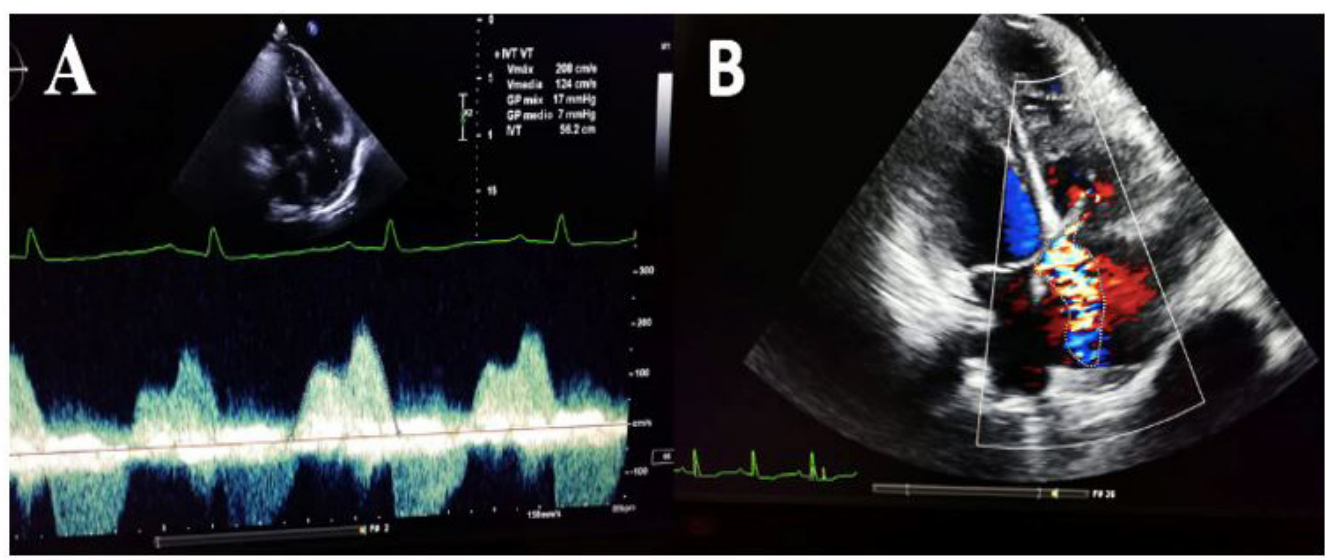

Figura 1. Doble lesión valvular tricúspidea. A. Figura de Doppler continuo que evidencia estenosis tricuspídea severa con gradiente máximo de $17 \mathrm{mmHg}$ y gradiente medio de 7 $\mathrm{mmHg}$, con área valvular por tiempo de hemipresión de $1.0 \mathrm{~cm} 2$. B: Doppler color en el que se evidencia insuficiencia de la válvula tricuspídea moderada a severa.

La patogénesis de la enfermedad cardíaca carcinoide es compleja y parcialmente entendida, debido a la gran cantidad de sustancias vasoactivas secretadas por el tumor: serotonina (5-HT), prostaglandinas, histamina, bradiquinina, factor de crecimiento transformante beta y taquiquininas (substancia $P$, neuroquinina $A$, neuropéptido $\mathrm{K})$, entre otras.

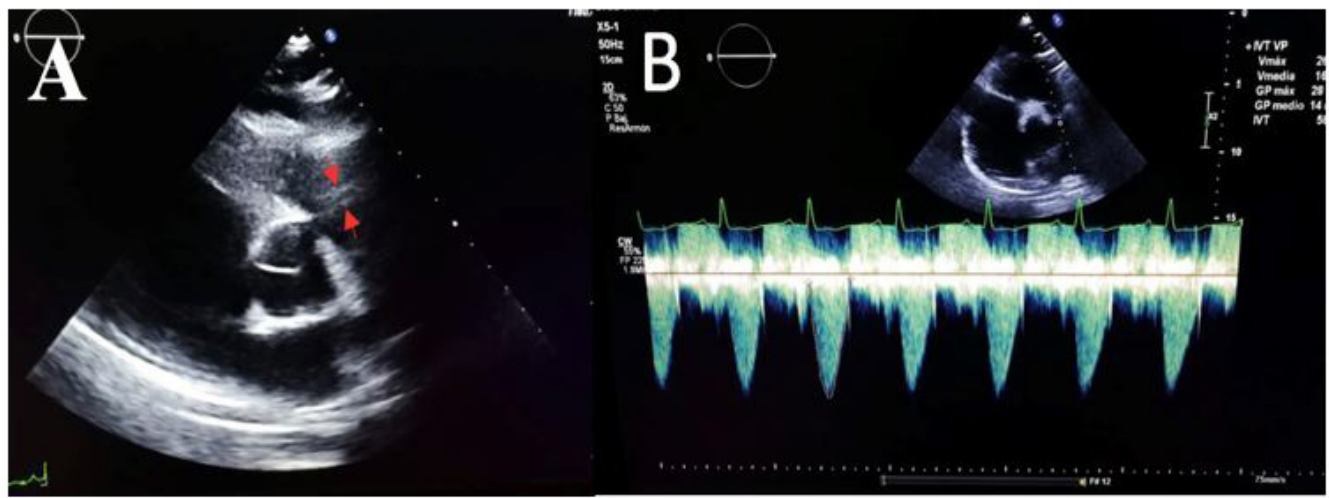

Figura 2. A. Válvula pulmonar con engrosamiento de sus valvas, estenosis leve, sin insuficiencia. B. Doppler continuo a través de la válvula pulmonar que evidencia estenosis leve con gradiente máximo de $28 \mathrm{~mm} / \mathrm{Hg}$.

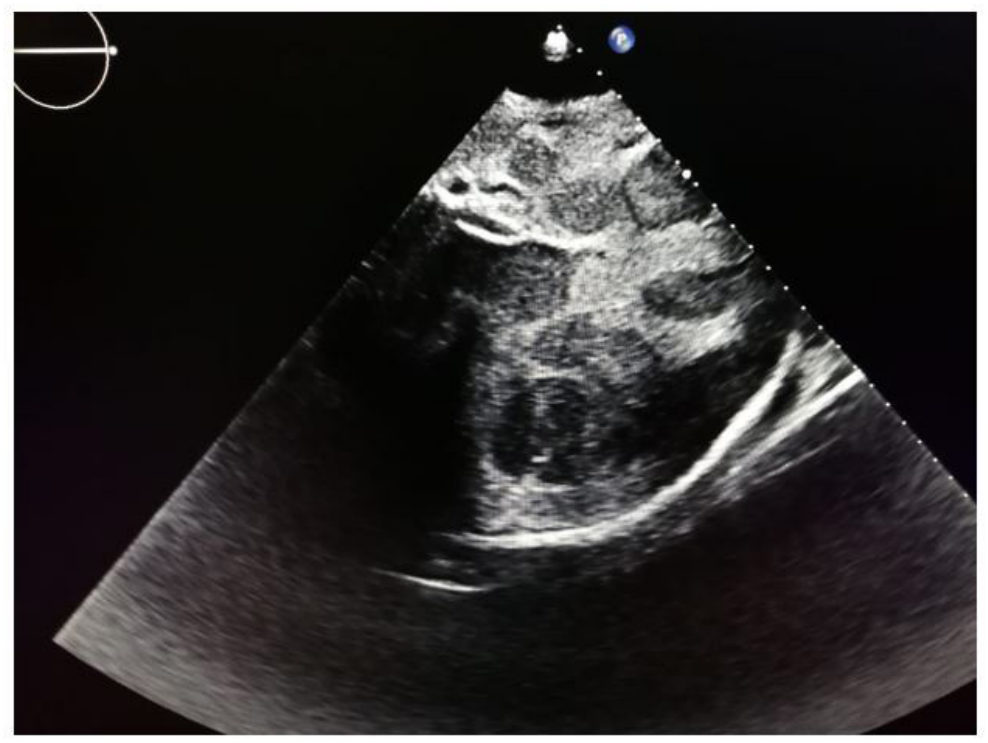

Figura 3. Vista ecocardiográfica subcostal modificada que evidencia múltiples imágenes nodulares hepáticas correspondientes a metástasis. 
Aunque es más frecuente el compromiso de cavidades cardíacas derechas debido a que las diferentes sustancias son degradadas y metabolizadas en hígado y pulmón, también se han reportado casos de compromiso izquierdo, tales como comunicaciones interauriculares o interventriculares con cortocircuito derecha a izquierda, tumores carcinoides bronquiales o niveles extremadamente altos de sustancias vasoactivas.

\section{Discusión}

A pesar de que los tumores neuroendocrinos tienen una baja prevalencia en la población general, hasta el 50 \% de ellos tendrán compromiso cardiovascular en algún momento y un 20 \% debutan con el mismo. El tener compromiso cardíaco aumenta la morbilidad y mortalidad asociadas al diagnóstico, dando una importancia adicional a su identificación. Sin tratamiento el pronóstico es pobre, con una sobrevida a tres años tan baja como del $31 \%$, comparado con $68 \%$ en los pacientes sin compromiso cardíaco $(5,8)$.

La patogénesis de la enfermedad cardíaca carcinoide es compleja y parcialmente entendida, debido a la gran cantidad de sustancias vasoactivas secretadas por el tumor: serotonina (5-HT), prostaglandinas, histamina, bradiquinina, factor de crecimiento transformante beta y taquiquininas (substancia $P$, neuroquinina $A$, neuropéptido K), entre otras (1-3). Sin duda, la más estudiada es la serotonina, teniendo una mayor importancia los receptores $5-\mathrm{HT}_{2 b^{\prime}}$ que se expresan en gran cantidad en las válvulas cardíacas. Estos receptores se encargan de estimular propiedades mitogénicas en los fibroblastos y células del músculo liso (serán los componentes de las placas fibrosas manifestadas en la enfermedad), así como la liberación de citoquinas proinflamatorias (2).

El resultado de este proceso a nivel celular, da como resultado el depósito de placas de material fibroso en la superficie endocárdica de las valvas, el aparato subvalvular, las diferentes cámaras cardíacas e incluso, en arterias pulmonares y aorta, y menos frecuentemente en vena cava (9).

Es más frecuente el compromiso de cavidades cardiacas derechas debido a que las diferentes sustancias son degradadas y metabolizadas en hígado y pulmón, incluyendo el transportador $5-\mathrm{HT}$, impidiendo su paso a las cavidades izquierdas (solo el $15 \%$ de todo los casos) $(10,11)$. No obstante, se ha reportado compromiso de cavidades izquierdas en casos específicos, tales como presencia de comunicaciones interauriculares o interventriculares con cortocircuito derecha a izquierda, tumores carcinoides bronquiales o niveles extremadamente altos de sustancias vasoactivas que sobrepasen la capacidad de degradación del hígado o el pulmón (12).

El diagnóstico siempre será un reto y dependerá de la alta sospecha clínica. Síntomas inespecíficos como fatiga, disnea y la presencia de edema deben aumentar su sospecha y la auscultación cardíaca puede denotar la presencia de un soplo valvular de novo $(13,14)$.

Algunos marcadores sanguíneos como el péptido natriurético son de gran ayuda en el abordaje inicial. Más no necesarios y pudieran ser más selectivos a la hora de definir la solicitud de una ecocardiografía transtorácica. Otros marcadores como la cromogranina A, la activina A o el ácido 5 hidroxiindolacético (5-HIAA, por sus siglas en inglés), este último medido en pacientes con diarrea y otros síntomas gastrointestinales, son usados dentro del abordaje de la enfermedad y como marcadores de progresión a compromiso cardiovascular. La medición urinaria de 5-HIAA se ve afectada por alimentos y medicamentos ingeridos, entre la aspirina, razón por la que la interpretación debe realizarse cuidadosamente (15-17).

Las imágenes diagnósticas son una herramienta fundamental, siendo la ecocardiografía transtorácica uno de los métodos de elección, donde los hallazgos pueden ser tan incipientes como un engrosamiento leve de una valva cardíaca (pudiendo 
Una vez establecido el diagnóstico y el compromiso cardiovascular debe instaurarse prontamente el tratamiento y definir la necesidad de recambio valvular quirúrgico. Compensar y manejar medicamente la falla cardíaca derecha dependerá principalmente de diuréticos, combinando principalmente tiazidas con diuréticos de asa y antagonistas del receptor de aldosterona, sabiendo de antemano que depletar de volumen al paciente sería contraproducente. pasar desapercibido) hasta engrosamiento marcado con parálisis de todas las valvas, generando una disfunción valvular severa. La insuficiencia tricuspídea leve es un hallazgo temprano; sin embargo, debe diferenciarse de otras etiologías. El compromiso carcinoide, entonces, se caracteriza por engrosamiento y retracción de las valvas, reduciendo su movilidad, causando pérdida de la curvatura normal y rectificación y alterando su movimiento principalmente en diástole $(10,18,19)$.

La resonancia magnética o la tomografía cardíaca pueden ser útiles para establecer el diagnóstico, usualmente cuando los hallazgos en la ecocardiografía no son concluyentes (mala ventana acústica) (10).

Estudios funcionales como el octreoscan o el PET con galio 68 no tienen un papel tan importante en la enfermedad cardíaca carcinoide, como si lo tienen en la búsqueda del primario del tumor primario neuroendocrino. La explicación está en la fisiopatología, pues las lesiones cardíacas son fibrosas y funcionalmente inactivas, razón por lo que estas pruebas no serían positivas. En los casos particulares donde existan metástasis cardíacas, se pudiera obtener un resultado positivo con los estudios funcionales $(3,18)$.

Una vez establecido el diagnóstico y el compromiso cardiovascular se debe instaurar prontamente el tratamiento y definir la necesidad de recambio valvular quirúrgico. Compensar y manejar medicamente la falla cardíaca derecha dependerá principalmente de diuréticos, combinando principalmente tiazidas con diuréticos de asa y antagonistas del receptor de aldosterona, sabiendo de antemano que depletar de volumen al paciente sería contraproducente $(2,3)$.

Pocas terapias farmacológicas tienen evidencia fuerte que sustente su uso en compromiso ventricular derecho; sin embargo, se deben considerar y siempre individualizar la necesidad de inhibidores de la enzima convertidora de angiotensina, bloqueadores del receptor de angiotensina, beta bloqueadores, digoxina o vasodilatadores.(13,20) Al mismo tiempo, se deben controlar los síntomas carcinoides con análogos de somatostatina como fármacos de primera línea.

Los tratamientos de segunda línea como interferón alfa, everolimus, quimio-embolización o cirugías citorreductoras, deben ser definidos según cada uno de los casos, llevando un manejo multidisciplinario entre Cardiología, Endocrinología y Cirugía endocrina (21).

En casos avanzados, refractarios a la terapia médica y en quienes el compromiso valvular severo contraindique procedimientos quirúrgicos (cirugías hepáticas), la cirugía cardiovascular es una opción (22-25). La decisión entre bioprótesis o prótesis mecánica debe ser individualizada; sin embargo, debido al alto riesgo de sangrado (aún más ante la presencia de metástasis hepáticas), las bioprótesis son más usadas y recientemente, el reemplazo valvular percutáneo comienza a mostrarse como una opción adicional $(4,26)$.

\section{Conclusión}

Los tumores neuroendocrinos son entidades poco prevalentes; sin embargo, por su alta incidencia de compromiso cardiovascular, es deber del cardiólogo conocerlo, diagnosticarlo e instaurar un tratamiento médico oportuno inicialmente y quirúrgico en caso de requerirlo. Por sus manifestaciones poco específicas, se debe mantener una alta sospecha clínica por parte del especialista y apoyarse de los hallazgos eco- 
No es infrecuente requerir reemplazo valvular quirúrgico, en cuyos casos por alto riesgo de sangrado, se prefiere el uso de bioprótesis sobre válvulas mecánicas. cardiográficos más característicos. El tratamiento y seguimiento se sugiere que sea por un equipo multidisciplinario. Existe poca evidencia para el tratamiento de la falla cardíaca derecha; sin embargo, según el compromiso valvular de cada paciente, el tratamiento debe basarse en diuréticos principalmente. No es infrecuente requerir reemplazo valvular quirúrgico, en cuyos casos por alto riesgo de sangrado, se prefiere el uso de bioprótesis sobre válvulas mecánicas.

\section{Fuente de financiación}

Ninguna.

\section{Conflictos de interes}

Se declara que no existe ningún conflicto de interés.

\section{Ética de la publicación}

Reporte de caso clínico que no incluye experimentación con animales ni ensayos clínicos. Todos los datos mostrados en las imágenes se recogen en la descripción del caso clínico y en la discusión.

\section{Bibliografía}

1. Bhattacharyya S, Davar J, Dreyfus G, Caplin ME. Carcinoid Heart Disease. Circulation. 2007:116(24):2860-5.

2. Paredes $A$, Valdebenito $M$, Neira $V$, Castro $P$, Zalaquett R. Síndrome carcinoide y compromiso valvular cardíaco. Rev Chil Cardiol. 2014;33(1):38-43.

3. Davar J, Connolly HM, Caplin ME, Pavel M, Zacks J, Bhattacharyya S, et al. Diagnosing and managing carcinoid heart disease in patients with neuroendocrine tumors. J Am Coll Cardiol. 2017;69(10):1288-304.

4. Shah PM. Valvulopatía tricuspídea y pulmonar: evaluación y tratamiento. Rev Esp Cardiol. 2010;63(11):1349-65.

5. Møller JE, Pellikka PA, Bernheim AM, Schaff HV, Rubin J, Connolly HM. Prognosis of carcinoid heart disease: analysis of 200 cases over two decades. Circulation. 2005;112(21):3320-7.

6. Hassan SA, Banchs J, Iliescu C, Dasari A, Lopez-Mattei J, Yusuf SW. Carcinoid heart disease. Heart. 2017;103(19):1488-95.

7. Perry D, Hayek SS. Carcinoid Heart Disease. Cardiol Clin. 2019;37(4):497-503.

8. Møller JE, Connolly HM, Rubin J, Seward JB, Modesto K, Pellikka PA. Factors associated with progression of carcinoid Heart Disease. N Engl J Med. 2003:348(11):1005-15.

9. Simula DV, Edwards WD, Tazelaar HD, Connolly HM, Schaff HV. Surgical Pathology of Carcinoid Heart Disease: A Study of 139 Valves From 75 Patients Spanning 20 Years. Mayo Clin Proc. 2002;77(2):139-47.

10. Bhattacharyya S, Toumpanakis C, Burke M, Taylor AM, Caplin ME, Davar J. Features of carcinoid heart disease identified by 2- and 3-dimensional echocardiography and cardiac MRI. Circ Cardiovasc Imaging. 2010;3(1):103-11. 
11. Maria Alegret J, Subirana MT, Marcuello E, Bayés-Genís A, Ferrés P, Torner M. Afectación valvular tricuspídea y pulmonar en un caso de síndrome carcinoide. Rev Esp Cardiol. 1997;50(7):532-4.

12. Connolly HM, Schaff HV, Mullany CJ, Rubin J, Abel MD, Pellikka PA. Surgical management of left-sided carcinoid heart disease. Circulation. 2001;104:I-36-I-40.

13. Fox DJ. Carcinoid heart disease: presentation, diagnosis, and management. Heart. 2004;90(10):1224-8.

14. Luis SA, Pellikka PA. Carcinoid heart disease: Diagnosis and management. Best Pract Res Clin Endocrinol Metab. 2016;30(1):149-58.

15. Korse CM, Taal BG, de Groot CA, Bakker RH, Bonfrer JMG. Chromogranin-A and $\mathrm{N}$-Terminal pro-brain natriuretic peptide: an excellent pair of biomarkers for diagnostics in patients with neuroendocrine tumor. J Clin Oncol. 2009;27(26):4293-9.

16. Bhattacharyya S, Toumpanakis C, Chilkunda D, Caplin ME, Davar J. Risk Factors for the Development and Progression of Carcinoid Heart Disease. Am J Cardiol. 2011;107(8):1221-6.

17. Zuetenhorst JM, Bonfrer JMGM, Korse CM, Bakker R, van Tinteren H, Taal BG. Carcinoid heart disease: The role of urinary 5-hydroxyindoleacetic acid excretion and plasma levels of atrial natriuretic peptide, transforming growth factor-Beta and fibroblast growth factor. Cancer 2003;97(7):1609-15.

18. Pandya UH, Pellikka PA, Enriquez-Sarano M, Edwards WD, Schaff HV, Connolly HM. Metastatic carcinoid tumor to the heart: echocardiographic-pathologic study of 11 patients. J Am Coll Cardiol. 2002;40(7):1328-32.

19. Matshela MR. Cases in a series of carcinoid syndrome and carcinoid heart disease. Cardiovasc J Afr. 13 de septiembre de 2018;29(4):e1-7.

20. Ferrari A, Glasberg J, Riechelmann R. Carcinoid syndrome: update on the pathophysiology and treatment. Clinics 2018;73.

21. Steeds RP, Sagar V, Shetty S, Oelofse T, Singh H, Ahmad R, et al. Multidisciplinary team management of carcinoid heart disease. Endocr Connect. 2019;8(12):R184-99.

22. Lillegard JB, Fisher JE, Mckenzie TJ, Que FG, Farnell MB, Kendrick ML, et al. Hepatic Resection for the carcinoid syndrome in patients with severe carcinoid heart disease: does valve replacement permit safe hepatic resection? J Am Coll Surg. 2011;213(1):130-6.

23. Mujtaba SS, Clark S. Carcinoid Heart Disease: Early outcomes after surgical valve replacement in nine patients. Heart Surg Forum. 16 de febrero de 2018;21(1):040.

24. Bonou M, Kapelios CJ, Kaltsas G, Perreas K, Toutouzas K, Barbetseas J. Cardiac Surgery for carcinoid heart disease: a weapon not to be misused. Cardiology. 2017;136(4):243-51. 
25. Mujtaba SS, Clark S. Quadruple Valve replacement for carcinoid heart disease. Braz J Cardiovasc Surg. 2018;33(4).

26. Heidecker B, Moore P, Bergsland EK, Merrick SH, Rao RK. Transcatheter pulmonic valve replacement in carcinoid heart disease. Eur Heart J - Cardiovasc Imaging. 2015. 